\title{
Can the turbulent galactic dynamo generate large-scale magnetic fields?
}

\author{
Kandaswamy Subramanian \\ Astronomy Centre, University of Sussex, Falmer, Brighton BN1 9QH, UK. \\ Princeton University Observatory, Peyton Hall, Princeton, NJ 08544. USA \\ NCRA, TIFR, Poona University Campus, Ganeshkind, Pune 41100\%. India
}

\begin{abstract}
Large-scale magnetic fields in galaxies are thought to be generated by a turbulent dynamo. However the same turbulence also leads to a small-scale dynamo which generates magnetic noise at a more rapid rate. The efficiency of the large-scale dynamo depends on how this noise saturates. We examine this issue taking into account ambipolar drift, which obtains in a galaxy with significant neutral gas. We argue that, (1) the small-scale dynamo generated field does not fill the volume, but is concentrated into intermittent rope like structures. The flux ropes are curved on the turbulent eddy scales. Their thickness is set by the diffusive scale determined by the effective ambipolar diffusion; (2) For a largely neutral galactic gas, the small-scale dynamo saturates, due to inefficient random stretching, when the peak field in a flux rope has grown to a few times the equipartition value; (3) The average energy density in the saturated small-scale field is sub equipartition, since it does not fill the volume; (4) Such fields neither drain significant energy from the turbulence nor convert eddy motion of the turbulence on the outer scale into wavelike motion. The diffusive effects needed for the large-scale dynamo operation are then preserved until the large-scale field itself grows to near equipartition levels.
\end{abstract}

Key words: Magnetic fields; turbulence; Galaxies:magnetic fields; ISM:magnetic fields; Cosmology: miscellaneous

\section{INTRODUCTION}

The origin of ordered, large-scale galactic magnetic fields remains a challenging problem. Magnetic fields in galaxies have strengths of order $10^{-6} G$, and are coherent on scales of several $\mathrm{kpc}$ (Beck et al. 1996). These fields can arise, in principle, by dynamo generation, from a weak, but nonzero, seed field $\sim 10^{-19}-10^{-23} G$, if the galactic dynamo can operate efficiently enough to exponentiate the field by a factor 30 - 40 (cf. Moffat 1978, Parker 1979, Zeldovich et al. 1983). However the capacity of presently known turbulent dynamo mechanisms to produce the observed galactic fields has been debated (Cattaneo and Vainshtein 1991, Vainshtein and Rosner 1991, Kulsrud and Anderson 1992, Brandenberg 1994).

It has been argued that magnetic noise in the form of small-scale fields, builds up much faster than the mean field in a turbulent flow. Magnetic noise can result from

\footnotetext{
* On leave from National Centre for Radio Astrophysics, TIFR, Poona University Campus, Ganeshkind, Pune 411007. India. email: ksub@astr.maps.susx.ac.uk, kandu@gmrt.ernet.in
}

the tangling of the large-scale field by the turbulence, or the operation of a small-scale dynamo. The dominant source, when one starts from weak large-scale seed fields, is the operation of a small-scale dynamo. Turbulence with a large enough magnetic Reynolds number (MRN), even when mirror-symmetric on average, generically leads to an exponential growth of fields correlated on the turbulent eddy scales, independent of the large-scale field. This growth occurs on the the turbulent eddy turnover timescale which is much smaller than the time scale for the growth of the mean field. Therefore the kinematic dynamo paradigm will become invalid long before the large-scale field has grown anywhere near the observed levels. Kulsrud and Anderson (KA) (1992) reach the somewhat bold conclusion that the galactic field must be of primordial origin! Although there are a number of mechanisms to produce small seed magnetic fields (Rees 1987, 1994, Ratra 1992, Subramanian 1995, Subramanian, Narasimha and Chitre, 1995 and references therein ), there is as yet no compelling mechanism to produce a field, anywhere near that required by the primeval hypothesis.

This problem may disappear if the small-scale dynamo generated fields can saturate, due to non-linear back reac- 
tion effects, in a manner which does not destroy the ability of turbulent motions to amplify the large-scale field. We examine whether this can indeed happen. Ideally one has to consider the MHD dynamo, where both the induction equation for the magnetic field, and the Euler equation for the velocity field, are solved simultaneously in a self consistent fashion. However this is a formidable task at present, even for numerical simulation. In this paper we take a first look at this difficult non-linear problem, in a simpler fashion by isolating the ingredients needed for the small-scale dynamo action and investigating under what conditions these get suppressed.

Galaxies have a significant neutral gaseous component. As magnetic fields grow, the Lorentz force on the charged component will cause a slippage between it and the neutrals. Its magnitude is determined by the friction between the components due to ion-neutral collisions. This drift, called ambipolar drift (Mestel and Spitzer 1956, Spitzer 1978, Draine 1980, 1986, Zweibel 1986), is one important non-linear feedback on both the small and large-scale dynamos, by the generated magnetic field. In another paper (Subramanian 1997 ; Paper II, in preparation), we give a derivation of the equations for both mean field and the magnetic correlations, incorporating the effects of ambipolar drift. We also give there the solution of these equations in several contexts. Some pertinent results of this work will be quoted here, where needed. We will see below, that the presence of neutrals, may be very important, in leading to a saturated state for the small-scale dynamo, which preserves large-scale dynamo action.

In section 2, we begin by introducing the turbulent galactic dynamo. In section 3, we summarise the properties of the kinematic small-scale dynamo action in Kolmogorov type turbulence and point out the problem they raise. The influence of ambipolar drift on the small-scale dynamo is considered in section 4 . Section 5 considers the the back reaction effects and saturation of the small-scale dynamo, due to the Lorentz forces acting on the fluid as a whole. The last section contains a discussion and summary of our results. We argue that, in galaxies, magnetic noise generated by the small-scale dynamo, may indeed saturate in a fashion which preserves large scale dynamo action.

\section{THE TURBULENT GALACTIC DYNAMO}

Spiral galaxies are differentially rotating systems. The magnetic flux is to a large extent frozen into the fluid and so any radial component of the magnetic field will be efficiently wound up and amplified to produce a toroidal component of the field. But this only results in a linear amplification of the field: to obtain the observed galactic fields starting from small seed fields we must find a way to generate the radial components of the field in the galaxy from the toroidal one. If this can be done, the field can grow exponentially and one has a dynamo.

The standard picture involves the effects of cyclonic turbulence in the galactic gas. The galactic interstellar medium is assumed to be turbulent, due for example to the effects of supernovae randomly exploding in different regions. In a rotating, stratified (in density and pressure) medium, like a disk galaxy, such turbulence becomes cyclonic and acquires a net helicity. Isotropic and homogeneous turbulence with helicity, in the presence of a large-scale magnetic field, $\mathbf{B}_{0}$, leads to an extra electromotive force of the form $\mathbf{E}=\alpha \mathbf{B}_{0}-\eta_{t} \nabla \times \mathbf{B}_{0}$, where $\alpha$ depends on the helical part of the turbulence and $\eta_{t}$ is the turbulent diffusion which depends on the non helical part of the turbulent velocity correlation function (Krause \& Radler 1980, Moffat 1978, Parker 1979). It should be noted that both the alpha effect, and turbulent diffusion, depend crucially on the diffusive (random walk) property of fluid motion (cf. Field 1996). So, if for some reason (see below) the fluid motion becomes wavelike, then the alpha effect and turbulent diffusion will be suppressed (Chandran 1996).

The induction equation, with the extra turbulent component of the electric field, a prescribed large-scale velocity field, can have exponentially growing solutions for the largescale field. These have been studied extensively in the literature (cf.Ruzmaikin, Shukurov \& Sokoloff 1988, Mestel \& Subramanian 1991, Beck et al. 1996 for a recent review). It had been assumed in most earlier works that the turbulent velocities do not get affected by Lorentz forces - until the mean large-scale field builds up sufficiently. However, this does not turn out to be valid due to the more rapid build up of magnetic noise compared to the value of the mean field, a problem to which we now turn.

\section{THE KINEMATIC SMALL-SCALE DYNAMO AND THE PROBLEM OF MAGNETIC NOISE}

Split up the magnetic field, $\mathbf{B}=\mathbf{B}_{0}+\delta \mathbf{B}$, into a mean field $\mathbf{B}_{0}$ and a fluctuating component $\delta \mathbf{B}$. Here, the mean field, $\mathbf{B}_{0}=<\mathbf{B}>$, is defined either as a spatial average over scales larger than the turbulent eddy scales or, more correctly, as an ensemble average. Kazantsev (1968) was the first to show that, even purely mirror-symmetric turbulence, leads to dynamo amplification of the small-scale fluctuating fields, for a sufficiently large magnetic Reynolds number. Some of the subsequent work is summarised by Zeldovich et al. (1983). The statistical properties of the small-scale field are most clearly expressed in terms of the magnetic correlation function $M_{i j}(r, t)=<\delta B^{i}(\mathbf{x}, t) \delta B^{j}(\mathbf{y}, t)>$, where $r=|\mathbf{x}-\mathbf{y}|$. Small scale dynamo action is described by the evolution of the longitudinal component $M_{L}(r, t)=r^{i} r^{j} M_{i j} / r^{2}$, where $r^{i}=x^{i}-y^{i}$.

In the case when the turbulent velocity, say $\mathbf{v}_{T}$, has a delta function correlation in time (Markovian), it is relatively straightforward to derive the evolution equation for $M_{i j}$ (cf. Kazantsev 1968, Vainshtein \& Kichatinov 1986, Paper II). Suppose we also assume $\mathbf{v}_{T}$ to be an isotropic, homogeneous, Gaussian random velocity field with zero mean. Specify its two point correlation function by $<$ $v_{T}^{i}(\mathbf{x}, t) v_{T}^{j}(\mathbf{y}, s)>=T^{i j}(r) \delta(t-s)$, with

$T^{i j}(r)=T_{N N}\left[\delta^{i j}-\left(\frac{r^{i} r^{j}}{r^{2}}\right)\right]+T_{L L}\left(\frac{r^{i} r^{j}}{r^{2}}\right)+C \epsilon_{i j f} r^{f}$.

Here, $T_{L L}(r)$ and $T_{N N}(r)$ are the longitudinal and transverse correlation functions for the velocity field and $C(r)$ represents the helical part of the velocity correlations. (cf. Landau and Lifshitz 1987). If $\mathbf{v}_{T}$ is assumed to be divergence-free, then, 
$T_{N N}(r)=\frac{1}{2 r} \frac{\partial}{\partial r}\left(r^{2} T_{L L}(r)\right)$.

The evolution of $M_{L}$ is given by

$\frac{\partial M_{L}}{\partial t}=\frac{2}{r^{4}} \frac{\partial}{\partial r}\left(r^{4} \kappa \frac{\partial M_{L}}{\partial r}\right)+G M_{L}$,

where we have defined

$$
\begin{aligned}
\kappa & =\eta+T_{L L}(0)-T_{L L}(r), \\
G & =-4\left[\frac{d}{d r}\left(\frac{T_{N N}}{r}\right)+\frac{1}{r^{2}} \frac{d}{d r}\left(r T_{L L}\right)\right] .
\end{aligned}
$$

The term involving $\kappa$ in equation (3) represents the effects of diffusion. The diffusion coefficient includes the effects of microscopic diffusion $(\eta)$ and a scale-dependent turbulent diffusion, $\left(T_{L L}(0)-T_{L L}(r)\right)$. The term proportional to $G(r)$, allows for the rapid generation of magnetic fluctuations, through shearing action and the existence of a smallscale dynamo independent of the large-scale field. [ In the evolution equation for $M_{L}$ given above, we have neglected its coupling to the helical part of the magnetic correlation due to a scale-dependent alpha effect. In the galactic context this has a negligible effect. We have also neglected the subdominant coupling to the large-scale field (see Paper II).]

Suppose $V$ and $L$ are the velocity and correlation lengths of the dominant energy carrying eddies of the turbulence, which is assumed to have a Kolmogorov energy spectrum. For numerical estimates we generally take $V=10 \mathrm{kms}^{-1}$ and $L=100 \mathrm{pc}$. For Kolmogorov turbulence, the eddy velocity at any scale $l$, is $v_{l} \propto l^{1 / 3}$, in the inertial range. The turbulence is cut off at a scale, say $l_{c} \approx L R_{e}^{-3 / 4}$, where $R_{e}=V L / \nu$ is the fluid Reynolds number and $\nu$ is the kinematic viscosity. We will be considering a largely neutral galactic gas and for this $\nu$ is dominated by the neutral contribution. We take the neutral-neutral collision to be dominated by $\mathrm{H}-\mathrm{H}$ collisons with a cross section $\sigma_{H-H} \sim 10^{-16} \mathrm{~cm}^{-2}$, leading to a kinematic viscosity $\nu \sim v_{t h}\left(1 / n_{H} \sigma_{H-H}\right)$. For a thermal velocity $v_{t h} \sim 10 \mathrm{kms}^{-1}$ and a neutral hydrogen number density $n_{H} \sim 1 \mathrm{~cm}^{-3}$, we have $\nu \sim 10^{22} \mathrm{~cm}^{2} \mathrm{~s}^{-1}$, so

$R_{e}=\frac{V L}{\nu} \approx 3 \times 10^{4} V_{10} L_{100}$

where $V_{10}=\left(V / 10 \mathrm{kms}^{-1}\right)$ and $L_{100}=(L / 100 \mathrm{pc})$. The magnetic Reynolds number at the outer scale of the turbulence is defined to be $R_{m}=(V L / \eta)$. For the Spitzer value of the resistivity $\eta=10^{7}\left(T / 10^{4} K\right)^{-3 / 2} \mathrm{~cm}^{2} \mathrm{~s}^{-1}$, with turbulence parameters as above, $R_{m}=3 \times 10^{19}$. Since $v_{l} \propto l^{1 / 3}$, the magnetic Reynolds number associated with eddies of size $l$ scales as $R_{m}(l)=v_{l} l / \eta=R_{m}(l / L)^{4 / 3}$.

The evolution of $M_{L}(r, t)$ has been studied in detail by several authors (Zeldovich et al 1983 and references therein) for the case of when $T_{L L}(r)$ has a single scale. We also study in Paper II, using WKBJ technique, the evolution of $M_{L}(r, t)$ for a model Kolmogorov type turbulence. We look at the properties of exponentially growing modes, $M_{L}(r, t)=M_{n}(r) e^{\Gamma_{n} t}$, which are regular at the origin, and tend to zero as $r \rightarrow \infty$. Some results of this work and the earlier works of Kazantsev (1968) and Zeldovich et al. (1983) pertinent to the present context, are summarised here.

- There is a critical value for the magnetic Reynolds number $(\mathrm{MRN}): R_{m}=R_{c} \approx 60$, for the excitation of the smallscale dynamo. Above this critical MRN the small-scale dy- namo can lead to an exponential growth of the fluctuating field correlated on a scale $L$. Further, the equations determining $R_{c}$ are the same if we replace $\left(L, R_{m}\right)$ by $\left(l, R_{m}(l)\right)$. Therefore, the critical MRN for excitation of a mode concentrated around $r \sim l$ is also $R_{m}(l)=R_{c}$, as expected from the scale invariance in the inertial range.

In the galactic context $R_{m}>>R_{c}$; in fact, one also has $R_{m}\left(l_{c}\right)=v_{c} l_{c} / \eta=R_{m} / R_{e}>>1$. (Here $v_{c}$ is the eddy velocity at the cut-off scale). Hence, small-scale dynamo action excites modes correlated on all scales from the cut-off scale $l_{c}$ to the external scale $L$ of the turbulence.

- Due to small-scale dynamo action, the fluctuating field, tangled on a scale $l$, grows exponentially on the corresponding eddy turnover time scale, with a growth rate $\Gamma_{l} \sim v_{l} / l$. Since $v_{l} \sim l^{1 / 3}$, the growth rate is $\Gamma_{l} \propto l^{-2 / 3}$, and so increases with decreasing $l$. In the galactic context, with $R_{m}\left(l_{c}\right)=R_{m} / R_{e}>>R_{c}$, the small-scale fields tangled at the cutoff scale grow more rapidly than any of the large-scale modes.

- The WKBJ analysis gives a growth rate $\Gamma_{c}=$ $\left(v_{c} / l_{c}\right)\left[5 / 4-c_{0}\left(\ln \left(R_{m} / R_{e}\right)\right)^{-2}\right]$ with $c_{0}=\pi^{2} / 12$ for the fastest mode. Also the growth rate for the small-scale dynamo is only weakly (logarithmically) dependent on $R_{m}$, provided $R_{m}$ is large enough.

- For the parameters adopted above, we have $\Gamma_{L}^{-1} \sim$ $L / V \sim 10^{7}$ yr. For the modes tangled at the cut-off scale, this time is $\Gamma_{c}^{-1} \sim 10^{4} \mathrm{yr}$. These times are much smaller than the time scale for the growth of the large-scale field.

The spatial structure of the dynamo-generated smallscale field is important in determining how the small-scale dynamo saturates. To examine the spatial structure for various eigenmodes of the small-scale dynamo, it is more instructive to consider the function $w(r, t)=\langle\delta \mathbf{B}(\mathbf{x}, t) \cdot \delta \mathbf{B}(\mathbf{y}, t)>$, which measures the correlated dot product of the fluctating field $\left(w(0)=<\delta \mathbf{B}^{2}>\right)$. Firstly there is a general constraint that can be placed on $w(r)$. Since the fluctuating field is divergence free, we have (Kleeorin et al. 1986, Paper II),

$w(r, t)=\frac{1}{r^{2}} \frac{d}{d r}\left[r^{3} M_{L}\right]$,

so

$\int_{0}^{\infty} w(r) r^{2} d r=\int_{0}^{\infty} \frac{d}{d r}\left[r^{3} M_{L}\right]=0$,

since $M_{L}$ is regular at the origin and vanishes faster than $r^{-3}$ as $r \rightarrow \infty$. Therefore the curve $r^{2} w(r)$ should have zero area under it. Since $w(0)=\left\langle(\delta \mathbf{B})^{2}\right\rangle, w$ is positive near the origin. And the fluctuating field points in the same direction for small separation. As one goes to larger values of $r$, there must then values of $r$, say $r \sim d$, where $w(r)$ becomes negative. For such values of $r$, the field at the origin and at a separation $d$ are pointing in opposite directions on the average. This can be intepreted as indicating that the field lines, on the average are curved on the scale $d$.

- In the case $R_{m} / R_{e}>>1, w(r)$ is strongly peaked within a region $r=r_{d} \approx l_{c}\left(R_{m} / R_{e}\right)^{-1 / 2}$ about the origin, for all the modes. Note that $r_{d}$ is the diffusive scale satisfying the condition $\eta / r_{d}^{2} \sim v_{c} / l_{c}$.

- For the most rapidly growing mode, $w(r)$ changes sign accross $r \sim l_{c}$ and rapidly decays with increasing $r / l_{c}$. 


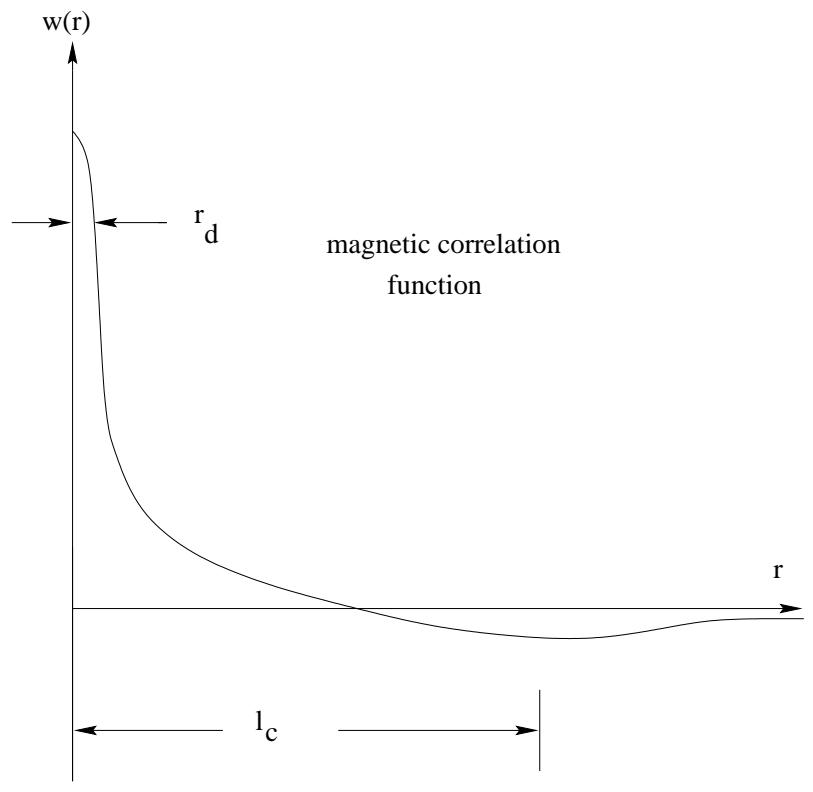

Fig. 1a interpretation as a

flux rope

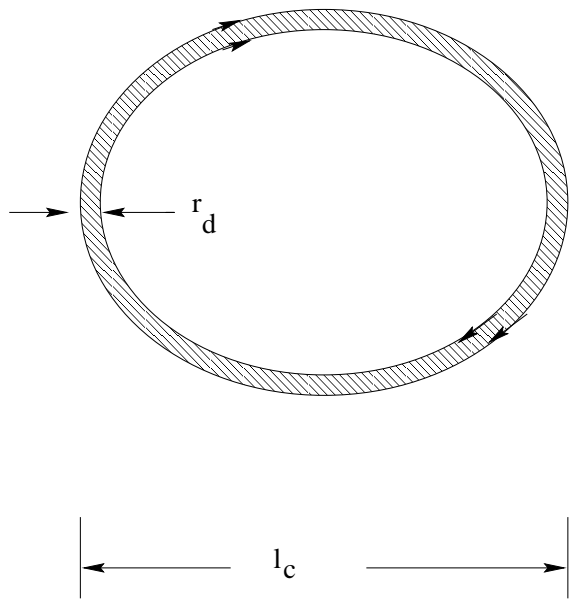

Fig. 1b

Figure 1. (a) Schematic representation of the the magnetic correlation function $w(r)$ for the fastest growing mode in the kinematic regime. The fact that $w(r)$ is positive at small $r$ and concentrated into a region of scale $r_{d}$ near the origin, implies that the field points in the same direction on the average for separations smaller than the diffusive scale $r_{d}$. The anti-correlation tail, with $w(r)<0$, is related to the vanishing of field divergence; that for points separated by the eddy scale $l_{c}$, the field points in opposite directions, on average. (b) The pictorial intepretation of $w(r)$ as a flux rope, with $r_{d}$ as the rope thickness and $l_{c}$ as curvature scale of the flux rope.

- For slower growing modes, with $\Gamma_{l} \sim v_{l} / l, w(r)$ extends up to $r \sim l$ after which it decays exponentially.

We should point out that a detailed analysis of the eigenfunctions, for the simple case when the longitudinal velocity correlation function has only a single scale, can be found in Kleeorin et. al. (1986). Their analysis is also applicable to the mode near the cut-off scale in Kolmogorov type turbulence. Further, these authors elaborate on a pictorial intepretation of the correlation function, in terms of the Zeldovich rope-dynamo (cf. Zeldovich et al. 1983). We have shown, schematically, $w(r)$ for the the fastest growing mode in Figure 1a. and its pictorial intepretation interms of a flux rope in Figure 1b. For the fastest growing mode there is only one node where $w(r)=0$. For higher order modes with smaller growth rates, several nodes for $w(r)$ will occur and can be intepreted interms of several scales on which the field is curved (cf. Kleeorin et al. 1986, see also Ruzmaikin et al. 1989 ). The extent of $w(r)$, (after which it decays exponentially), gives the largest scale on which the flux rope is curved.

- If one adopts this intepretation of the $w(r)$ for the various modes, the small-scale field can be thought of as being concentrated in rope like structures with thickness of order the diffusive scale $r_{d}<<l$ and curved on a scale up to $\sim l$ for modes extending up to $r \sim l$.

To end this section we give a qualitative picture of the mechanism for the dynamo growth of small-scale fields. When the field starts from an arbitrary initial configuration, in the kinematic stage, the initial field growth, is just due to random stretching by the turbulence, together with flux freezing. Eddies of scale $l$ stretch and tangle the field on corresponding scales. The field grows at this stage because the stretching of a "flux rope" leads to a decrease in its cross section (due to near incompressibility of the flow), and hence from flux freezing an increase in the field strength. As the field grows, the magnetic field gets concentrated into smaller and smaller scales until diffusion comes into play.

Consider to begin with the effect of eddies at the cutoff scale, $l_{c}$, of the turbulence. The initial amplification due to purely stretching of the field by these eddies stops when the field has been concentrated into a small enough scale $r_{d}$, such that the rate of diffusion across $r_{d}$ becomes comparable to the stretching rate by these eddies. This is when

$\eta / r_{d}^{2} \sim v_{c} / l_{c}$.

This gives $r_{d} \sim l_{c} / R_{m}^{1 / 2}\left(l_{c}\right)$. We will refer to $r_{d}$ as the thickness of the flux rope curved on a scale $l_{c}$. Further growth of the field can only be achieved by the operation of the smallscale dynamo, which exponentiates the field for a MRN above $R_{c}$. This is explicitly demonstrated by the solutions for the kinematic small-scale dynamo discussed above and can be thought of as the operation of the Zeldovich - StretchTwist-Fold - rope dynamo at random locations.

For galactic gas with $R_{m} / R_{e}>>1$, even the eddies at the cut-off scale have an MRN greater than the critical value needed for dynamo action. These eddies exponentiate the field at a growth rate $v_{c} / l_{c}$. Larger eddies of scale $l>l_{c}$ also lead to stretching, twisting and folding of the field at a slower rate, $v_{l} / l$. This leads to dynamo growth of fields tangled at scale $l$, with a slower growth rate $v_{l} / l$. The field curved on a scale $l$ is also, at the kinematic stage, chopped up further by smaller scale eddies (a scale dependent turbulent 
diffusion ) until its energy can be dissipated by microscopic diffusion at scale $r_{d}$. So, on any flux rope of length $l$, one has smaller scale wiggles until the diffusive scale $r_{d}$.

We emphasise that the time scale for mean-field growth is $\sim 10^{9} \mathrm{yrs}$, of order a few rotation time scales of the disk, and is much larger than the time scale for the growth of the fluctuating field ( $\Gamma_{L}^{-1} \sim 10^{7} \mathrm{yr}$ ). Hence, the operation of the small-scale dynamo will imply that the magnetic field is rapidly dominated by the fluctuating component. Chandran (1996) has shown that the presence of small-scale magnetic fields could change hydrodynamic turbulence into magnetoelastic waves, with a phase velocity $\bar{v}_{A}$, where $\bar{v}_{A}^{2}$ is two thirds of the magnetic energy per unit mass. So, if the energy density in the small-scale magnetic noise builds up to equipartition levels, the fluid motions could become predominantly wavelike, with a wave period of order the eddy turnover time. This could then lead to a reduced alpha effect and turbulent diffusion. Unless the build up of magnetic noise is curbed in a way which leaves the turbulence still having a diffusive property, large-scale dynamo action will be severely affected. We now turn to the effect of ambipolar drift and the possible ways in which the small-scale dynamo saturates.

\section{THE EFFECT OF AMBIPOLAR DRIFT}

In a partially ionised medium the magnetic field evolution is governed by the induction equation

$(\partial \mathbf{B} / \partial t)=\nabla \times\left(\mathbf{v}_{i} \times \mathbf{B}-\eta \nabla \times \mathbf{B}\right)$,

where $\mathbf{v}_{i}$ the velocity of the ionic component of the fluid. The ions experience the Lorentz force due to the magnetic field. This will cause them to drift with respect to the neutral component of the fluid. If the ion-neutral collisions are rapid enough, one can assume that the Lorentz force on the ions is balanced by their friction with the neutrals. Under this approximation, the Euler equation for the ions reduces to :

$\rho_{i} \nu_{i n}\left(\mathbf{v}_{i}-\mathbf{v}_{n}\right)=\frac{(\nabla \times \mathbf{B}) \times \mathbf{B}}{4 \pi}$,

where $\rho_{i}$ is the mass density of ions, $\nu_{i n}$ the ion-neutral collision frequency and $\mathbf{v}_{n}$ the velocity of the neutral particles.

The ion-neutral elastic scattering frequency is given by $\nu_{i n}=\rho_{n}\langle\sigma v\rangle /\left(m_{i}+m_{n}\right)$, where $\rho_{n}$ is the neutral fluid density, and $m_{i}, m_{n}$ are the ion and neutral particle masses (cf. Mestel and Spitzer 1956, Draine 1980, 1986). We will assume that the galaxy had very nearly primordial composition in its early stage of evolution: then the ions are mostly just protons and the neutrals are mostly hydrogen atoms. Elastic scattering occurs with the ion polarising the neutral atom, and interacting with the induced dipole. For $H-H^{+}$interactions, in addition to elastic scattering, there can also be charge exchange reactions, which increase the ion-neutral cross-section. Draine (1980) adopts the maximum of these two rates, and gives a momentum transfer rate coefficient of $\langle\sigma v\rangle \approx 3.2 \times 10^{-9} \mathrm{~cm}^{3} \mathrm{~s}^{-1}$ for $v<2 k m s^{-1}$, and $\left\langle\sigma v>\approx 2.0 \times 10^{-9}\left(v / \mathrm{kms}^{-1}\right)^{0.73} \mathrm{~cm}^{3} \mathrm{~s}^{-1}\right.$ for $2 \mathrm{kms}^{-1}<v<1000 \mathrm{kms}^{-1}$. In the galactic disk, we expect the gas to have a temperature $T<10^{4} \mathrm{~K}$ with $v \sim$ $10 \mathrm{kms}^{-1}$ and so $\langle\sigma v\rangle\left(H-H^{+}\right) \sim 10.74 \times 10^{-9} \mathrm{~cm}^{3} \mathrm{~s}^{-1}$. Further, the interaction with helium atoms will not give a significant addition to the collision rate because the polarisability of helium is less due to its symmetry and helium is 4 times heavier than hydrogen. However, helium will contribute about $25 \%$ of the total density of the fluid. Let $\rho_{H^{+}}$, $\rho_{H}$ be the proton and hydrogen densities and $n_{i}=\rho_{H+} / m_{H}$. We then have

$\left.\rho_{i} \nu_{i n}=\frac{\left.\rho_{H^{+}} \rho_{H}<\sigma v\right\rangle_{H-H^{+}}}{2 m_{H}}=n_{i} \rho_{n}<\sigma v\right\rangle_{\text {eff }}$

with $\langle\sigma v\rangle_{\text {eff }} \sim 4 \times 10^{-9} \mathrm{~cm}^{3} \mathrm{~s}^{-1}$.

For the evolution of the small-scale field, we show in Paper II, that ambipolar drift adds to the diffusion coefficient, $\kappa$, a term proportional to the energy density in the fluctuating fields. This changes $\eta$ to an effective value

$\eta_{a m b i}=\eta+\frac{w(0, t)}{6 \pi \rho_{i} \nu_{i n}}=\eta+\frac{<\delta \mathbf{B} \cdot \delta \mathbf{B}>}{6 \pi \rho_{i} \nu_{i n}}$,

and replaces $\kappa$ in equation (3) for $M_{L}(r, t)$, by a new $\kappa_{N}=\eta_{a m b i}+T_{L L}(0)-T_{L L}(r)$. One can define an effective magnetic Reynolds number, incorporating the effect of ambipolar drift, for fluid motion on any scale of the turbulence by

$R_{a m b i}(l)=\frac{v_{l} l}{\eta_{a m b i}}=\frac{v_{l} l 6 \pi \rho_{i} \nu_{i n}}{<\delta \mathbf{B} \cdot \delta \mathbf{B}\rangle}$,

where $v_{l}=(l / L)^{1 / 3} V$ as before.

As the energy density in the fluctuating field increases, $R_{a m b i}(l)$ decreases. Firstly, this makes it easier for the field energy to reach the diffusive scales, from a general initial configuration. After this stage, the initial amplification due to purely stretching stops, and further growth of the field can only be achieved by the operation of the small-scale dynamo. If, as the field grows, $R_{a m b i}(L)$ decreases sufficiently, a stationary state with $\partial M_{L} / \partial t=0$ could, in principle, be achieved. In such a state, $M_{L}$ is independent of time. So, the condition on the critical MRN for the stationary state to be reached, will be identical to that obtained in the kinematic stage. That is, if $R_{a m b i}(L)$ decreases to a value $R_{c} \sim 100$, dynamo action will stop completely.

However, for galactic turbulence,

$R_{a m b i}(l)=\frac{1}{f(l)} \frac{3 \rho_{i} \nu_{i n} l}{2 \rho_{n} v_{l}}=\frac{Q(l)}{f(l)}$,

where $f(l)=B_{l}^{2} /\left(4 \pi \rho_{n} v_{l}^{2}\right)$ is the ratio of the local magnetic energy density of a flux rope curved on scale $l$, to the turbulent energy density $\rho_{n} v_{l}^{2} / 2$ associated with eddies of scale $l$. Using the value of $\nu_{i n}$ as determined in Eq. (11) and putting in numerical values we get

$Q(l)=\frac{3 \rho_{i} \nu_{i n} l}{2 \rho_{n} v_{l}} \sim 1.8 \times 10^{4} n_{-2}\left(\frac{l}{L}\right)^{2 / 3} L_{100} V_{10}^{-1}$

where $n_{-2}=\left(n_{i} / 10^{-2} \mathrm{~cm}^{-3}\right)$, and we have assumed a Kolmogorov scaling for the turbulent velocity fluctuations.

One can see from Eq. (14) - 15) that, for typical parameters associated with galactic turbulence, the MRN incorporating ambipolar drift is likely to remain much larger than $R_{c}$ for most scales of the turbulence, even when the field energy density becomes comparable to the equipartition value. So ambipolar drift by itself cannot saturate the small scale dynamo. Rather, one expects the field to continue to grow rapidly, even taking into account ambipolar drift. Note also that the growth rates for the small-scale dynamo generally depends only weakly on the MRN, provided the MRN is much larger than $R_{c}$. (see section 3, Kleeorin et al. 1986, Ruzmaikin et al. 1989). Therefore, we still expect 
the small-scale dynamo-generated field to grow almost exponentially on the eddy turn around time scale, as long as $R_{a m b i}>R_{c}$.

The spatial structure of the fluctuating field will also remain ropy, as argued in section 3 , as long as $R_{a m b i}>>R_{c}$. However, as the field strength in a flux rope, curved on a scale $>l$, grows to near equipartition with the turbulent energy associated with eddies smaller than $l$, these smaller scale eddies would no longer be effective in causing "turbulent diffusion" of the larger scale field. So the thickness $r_{d}(l)$ of a flux rope curved on a scale $l$ is determined eventually by demanding that the ambipolar diffusion timescale across $r_{d}(l)$ becomes comparable to the stretching timescale $l / v_{l}$; that is,

$r_{d}^{2}(l) / \eta_{a m b i} \sim l / v_{l}$.

This determines the thickness to be $r_{d}(l) \sim l / R_{a m b i}^{1 / 2}(l)$. Since $R_{a m b i}(l)>>1$, we expect flux ropes to remain relatively thin with a thickness $\sim l / R_{a m b i}^{1 / 2}(l)<<l$, even taking account of the ambipolar drift.

In summary, we have argued here that, in galaxies, the small-scale dynamo continues to exponentiate the field fluctuations even in the presence of ambipolar drift. This fluctuating field however does not fill the volume but is concentrated into intermittent rope like structures. The ropes are curved on the turbulent eddy scale and their thickness is set by the diffusive scale $r_{d}(l)$ determined by the effective ambipolar diffusion.

We have to consider how other non-linear feedback processes could limit the growth of this small-scale dynamo generated field.

\section{SATURATION OF THE SMALL-SCALE DYNAMO}

\subsection{Inefficient random stretching and damping}

The first of these restraining processes is the reduction in the efficiency of stretching of a flux rope as the field in the rope, say $B_{p}$, grows in strength. A turbulent eddy of scale $l$ produces a correlated winding up of the field for a time of order $l / v_{l}$. Note that this is just a consequence of the induction $\nabla \times(\mathbf{v} \times \mathbf{B})$ term and velocity shear. No dynamics is involved. However, suppose the growing tension component of the Lorentz force on the flux rope tangled on this scale, can untangle (or straighten) the rope and damp away its wrinkle, on a comparable timescale. The random stretching of the flux rope by these eddies will be suppressed and the small scale dynamo will not operate efficiently.

To estimate the straightening time-scale, say $t_{s}$, we have to look at the dynamics of the flux rope. The motion of the flux rope through the surrounding medium is influenced not only by the tension force but also by friction. (We are neglecting, for now, the effects of rotation and gravity). Suppose $v_{s}$ is the velocity associated with the untangling or straightening of the field in the flux rope. If one were to ignore the effect of friction, we would have $v_{s} \sim v_{A}=\left(B_{p}^{2} / 4 \pi \rho_{n}\right)^{1 / 2}$. However, the effect of drag leads to a smaller "terminal" velocity, which can be estimated by equating the tension force to the drag on the flux rope. Also, the work done by the rope against the frictional drag leads to a damping of the energy associated with the wrinkle of the flux rope on the straightening timescale, $l / v_{s}$, which is comparable to the rapid eddy turnover time when $v_{s} \sim v_{l}$. We illustrate schematically, this process of flux rope straightening and damping in Figure 2a.

The drag force per unit length on the flux rope is $\sim C_{d} \rho_{n} v_{s}^{2} r_{d} / 2$ (cf. Parker 1979 Eq. 8.59). The coefficient $C_{d}$ depends on the fluid Reynolds number on the scale of the radius of the flux rope, that is on $R_{e}^{r a d}=v_{s} r_{d} / \nu$. Note that the drag formalism is not well developed for the case when the external medium is itself turbulent (cf. Parker 1979 Section 8.7). One expects eddies with a scale smaller than the rope radius to have a different effect compared to larger eddies. In our case, $r_{d}<<l$, and so one expects momentum transfer by eddies with scale $<r_{d}$ to be subdominant compared to the turbulent drag induced by larger eddies. Here we adopt the drag formula given above, with the assumption that the complications mentioned above do not drastically change the results.

Equating the magnetic tension component of the Lorentz force in the rope and drag then gives

$\frac{B_{p}^{2}}{4 \pi(l / 2)}\left(\pi r_{d}^{2}\right) \sim \frac{C_{d}}{2} \rho_{n} v_{s}^{2} r_{d}$

Here, we have taken the curvature radius associated with the field tangled on scale $l$ to be $l / 2$. The dynamo action on these scales will be affected when $t_{s} \sim l / v_{s} \sim l / v_{l}$ or when $v_{s} \sim v_{l}$. Since, in this case, the flux rope is able to straighten and damp (due to friction) its wrinkle (curvature) on a timescale comparable to the stretching timescale $l / v_{l}$. So, the random stretching of the field by eddies of scale $l$, will become inefficient when the peak field has grown to a value found by substituting $v_{s} \sim v_{l}$ in Eq. (17). Straight forward rearrangment of the various quantities in Eq. (17 ) then implies an upper limit on the magnetic field in the flux rope, of

$f_{p}(l)=\frac{B_{p}^{2} / 8 \pi}{\left(\rho_{n} v_{l}^{2} / 2\right)} \approx \frac{C_{d}}{4 \pi} \frac{l}{r_{d}}$.

Since we expect $r_{d}<l$, the peak field in the rope can exceed equipartition values.

When ambipolar drift is causing the flux rope to thicken, we have $l / r_{d}=R_{a m b i}^{1 / 2}(l)$, which itself depends on the magnetic field strength in the rope. Further, the drag co-efficient, $C_{d}$, depends on $R_{e}^{r a d}$, so on $r_{d}$, and therefore implicitly on the field in the rope.

The value of $C_{d}$ decreases from about 30 at $R_{e}^{\text {rad }} \sim 1$ to 4 at $R_{e}^{r a d} \sim 10,1$ at $R_{e}^{r a d} \sim 10^{2}$ and ranges between $1-0.1$ for larger values (cf. Parker 1979 Sec.8). For our problem, an adequate estimate of the drag co-efficient is obtained by taking $C_{d} \sim 10 / \sqrt{R_{e}^{r a d}\left(r_{d}\right)}$. Taking $v_{s} \sim v_{l}$, we have $R_{e}^{r a d}=$ $R_{e}(l)\left(r_{d} / l\right)$. Using $l / r_{d}=R_{a m b i}^{1 / 2}(l)$, and re-arranging, we obtain

$$
\begin{aligned}
f_{p}(l) & \approx\left(\frac{10}{4 \pi}\right)^{4 / 7} \frac{Q^{3 / 7}(l)}{R_{e}^{2 / 7}(l)} \\
& \approx 3.1 n_{-2}^{3 / 7}\left(\frac{l}{L}\right)^{-2 / 21} L_{100}^{1 / 7} V_{10}^{-5 / 7}
\end{aligned}
$$

One can now go back and estimate the Reynolds number $R_{e}^{r a d}$. We get

$R_{e}^{\text {rad }} \sim 392(l / L)^{19 / 21} n_{-2}^{-2 / 7} V_{10}^{3 / 2} L_{100}^{1 / 2}$. 


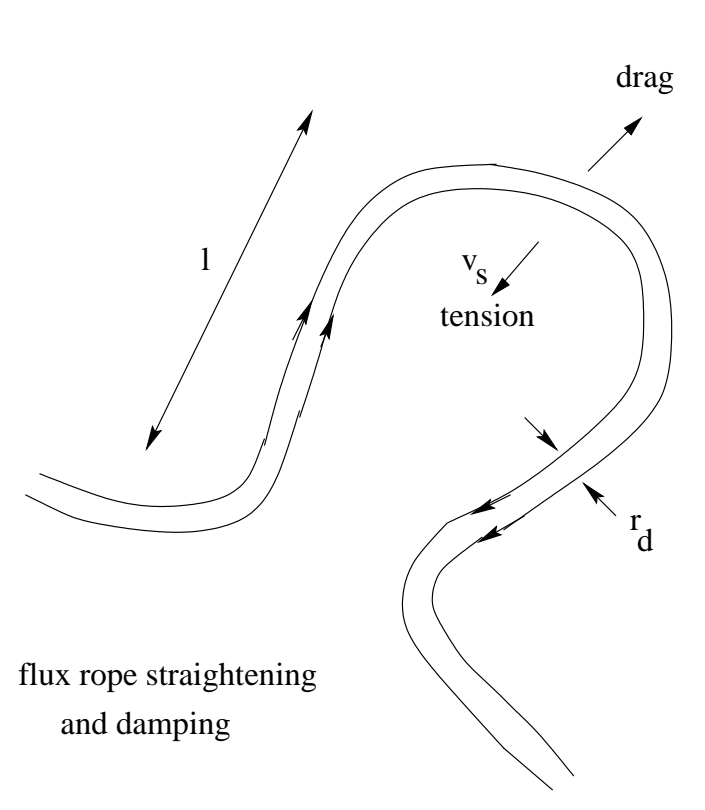

Fig. 2a

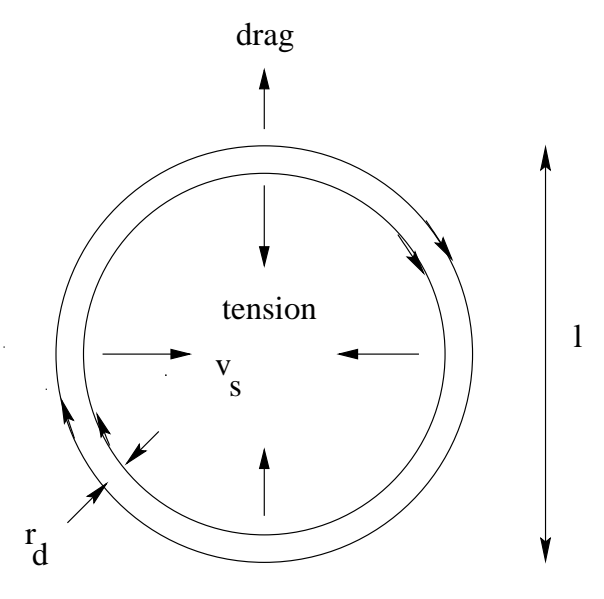

flux loop collapse

Fig. $2 b$

Figure 2. (a) A schematic illustration of a curved flux rope, with a radius of curvature $l$ and thickness $r_{d}$. Magnetic tension acts to straighten the rope and aerodynamic drag damps the magnetic energy associated with the wrinkle in the rope. This leads to inefficient random stretching when $v_{s}$ becomes comparable to $v_{l}$. (b) The collapse of flux loops is illustrated schematically. Such collapse results in an irreversible removal of small-scale magnetic noise and limits the value of $N$.

At the cut off scale $l_{c}, R_{e}^{r a d} \sim 0.4$, while at the outer scale $L, R_{e}^{r a d} \sim 392$. So the approximation used for the drag coefficient should be reasonably accurate.

We see from Eq. (19), that when the field in the flux rope of scale $l$ grows to a few times the equipartition field associated with eddies on that scale, the random stretching of the rope will become inefficient, limiting its further growth by dynamo action. Since larger eddies carry larger energy, this also implies that the the saturated value of the field in a flux rope, tangled on a scale $l$ will be larger for larger $l$.

The average energy density in the field tangled on the scale $l$, however need not exceed the energy density of the turbulent eddies because of the ropiness of the field. Since the ropes have a thickness of order $r_{d}$ and are curved an a scale $l$, the average energy density contributed by a flux rope to a sphere of radius $l$ is

$E_{B}(l) \sim \frac{B_{p}^{2}}{8 \pi} \frac{\pi r_{d}^{2} \pi l}{(4 \pi / 3) l^{3}}=\frac{B_{p}^{2}}{8 \pi} \frac{3 \pi}{4} \frac{r_{d}^{2}}{l^{2}}$.

Note that, although $B_{p}^{2} \propto\left(l / r_{d}\right)$, and can exceed equipartition value, the average energy density of a flux rope in a correlation volume $E_{B}(l) \propto B_{p}^{2}\left(r_{d} / l\right)^{2} \propto\left(r_{d} / l\right)$. This can be much smaller than equipartition because $r_{d} / l<<1$.

In principle, the length of the flux rope tangled on a scale $l$ may be larger than the value $\pi l$ assumed above, and equal to say $N \pi l$. In this case, the ratio of the average magnetic energy density of the field tangled on scale $l$ to the turbulent energy on the same scale is

$F(l)=\frac{E_{B}(l)}{\left(\rho_{n} v_{l}^{2} / 2\right)} \sim N\left(\frac{3 C_{d}}{16}\right)\left(\frac{r_{d}}{l}\right)$.

Putting in numerical values, we have
$F(l)=0.9 \times 10^{-3} N n_{-2}^{-1 / 4}\left(\frac{l}{L}\right)^{-5 / 6} L_{100}^{-3 / 4} V_{10}^{-1 / 4}$

We can also ask if the energy dissipated in ambipolar drift and frictional drag is comparable to the turbulent power in the case when the small scale field saturates due to the stretching constraint. Since the ambipolar drift rate across the rope is comparable to the stretching rate, $v_{l} / l$, the power dissipated by ambipolar drift is $\left(\eta_{a m b i} / r_{d}^{2}(l)\right) E_{B}(l) \sim E_{B}(l)\left(v_{l} / l\right)$. The power lost due to frictional drag is also $\sim E_{B}(l)\left(l / v_{l}\right)$. So the ratio of the total power dissipated to the turbulent power is

$\frac{P_{D}}{P_{T}}=2 \frac{E_{B}(l)\left(v_{l} / l\right)}{\left(\rho_{n} v_{l}^{2} / 2\right)\left(v_{l} / l\right)} \sim 2 F(l)$

Both the energy density in the flux ropes, and the energy dissipated due to ambipolar drift and friction depend on how large $N$ can get, or how many flux ropes of scale $l$ are packed into a correlation volume of radius $l$, in the final saturated state.

\subsection{The limiting effect due to flux loop collapse}

An important process, which limits $N$ from becoming too large, enters as the peak field in the flux rope increases to the value given by Eq. 18. As one tries to pack more and more flux ropes curved on a scale $l$ into a volume of same scale, the probability that the rope intersects itself, or another co-habiting rope, increases. This will result in loops of flux of radius $l$. Also, note that the the very process by which the small-scale dynamo may operate, viz. via the stretchingtwisting-folding actions associated with the Zeldovich rope dynamo, will generically result in flux loops of scale $l$. 
These loops of flux, when not being stretched, can collapse to a small radius (cf. Deluca, Fisher \& Patten 1993, Vishniac 1995).This is schematically illustrated in Figure $2 \mathrm{~b}$. The time-scale for the collapse is of order $l / v_{s}$, which will be $\sim l / v_{l}$, the eddy turnover time, since $v_{s} \sim v_{l}$ when the peak field in the rope is given by Eq. (18). The collapse of the loop will be halted when it has shrunk to a radius comparable to its thickness. At this stage, the effects of diffusion could convert most of the remaining energy in the loop into kinetic energy and heat. This process results in the irreversible removal of energy from the small-scale magnetic field tangled on scale $l$ at a rate $v_{l} / l$, comparable to its rate of build up by turbulent stretching, after the field has grown sufficiently. A dynamical equilibrium for $\mathrm{N}$ can then result, whereby "old" loops of flux, which have peak flux given by Eq. (18), collapse and are destroyed at a rate $v_{l} / l$, to be replaced by newly created loops, at the same rate, which are just reaching the saturated value of the peak flux. This picture of dynamical equilibrium leads one to conjecture that, in the saturated state, the average length of flux ropes tangled on scale $l$, in a volume of radius $l$, cannot grow much larger than $\pi l$. That is the value of $N$, can not grow much larger than unity.

Given that $N$ is not too large, one can see from Eq. (24) that the power dissipated in ambipolar drift is much smaller than the turbulent power. This occurs for all scales except near the cut-off scale. In fact, one only needs $N$ less than about 100 for the eddies at the energy-carrying scale to be left unaffected (undamped) by the growth of the small-scale field. Also, from Eq. (23), one can see that the average energy density of the generated small-scale field is much smaller than the average energy density in the turbulence. So, any wave-like motion induced by the presence of the small-scale field (cf. Chandran 1996) will have a period larger than the eddy turn around time. This implies that such tangled small-scale fields do not change the diffusive nature of the turbulence. For these reasons, the large-scale dynamo can still operate to generate the mean field.

\subsection{The limit on the field in flux ropes due to the external pressure of the gas}

There is one caveat to the above discussion. We have assumed that the field in the rope can grow sufficiently so that the tension in the rope begins to play an important role in the rope dynamics. However, there is an upper limit to the growth of the magnetic field in the ropes, from the effect of its magnetic pressure on the dynamo process. Due to the increasing importance of this pressure, stretching of field lines can lead to a partial decrease in fluid density in the ropes rather than a decrease in the rope-cross section and the associated increase in the rope magnetic field (cf. Vishniac 1995). An upper limit to the magnetic pressure in the ropes is given by the external pressure $P_{e x t}$. This implies that the field in the rope, is limited to

$B_{p}<\left(8 \pi P_{\text {ext }}\right)^{1 / 2}$.

Whether the field in the ropes will be limited by inefficient random stretching as given by Eq. (19) or by external pressure (Eq. (25)) will depend on the parameters of the problem. The rope field will be given by the lower of the limiting fields implied by Eqs. (19) and (25).
The total pressure of the interstellar medium in a galaxy could contain a number of components; a thermal component, pressure due to turbulence itself and possibly due to non-thermal "cosmic- rays". The ratio of the gas pressure to the turbulent energy density is

$P_{g} / E_{T} \sim 1.7\left(T / 10^{4} K\right) V_{10}^{-2}$.

If $P_{\text {ext }}$ in a galaxy is a factor $F$ times the gas pressure, then the peak field given by Eq. (19) begins to exceed that given by Eq. (25), when the ion density exceeds a critical value $n_{i}^{c}$. This critical value is given by

$$
\begin{aligned}
n_{i}^{c} & \sim 6.4 \times 10^{-2}\left(\frac{n_{n}}{1 c m^{-3}}\right)^{2 / 3} \mathrm{~cm}^{-3} \\
& \times \quad V_{10}^{-3} L_{100}^{-1 / 3}\left(\frac{L}{l}\right)^{4 / 3}\left(\frac{F}{4}\right)^{7 / 3}\left(\frac{T}{10^{4} K}\right)^{7 / 3} .
\end{aligned}
$$

For a larger ion density than $n_{i}^{c}$, the peak field for the flux ropes tangled on the largest scale $L$ saturates to a value $B_{p} \sim\left(8 \pi P_{\text {ext }}\right)^{1 / 2}$, lower than given by Eq. (19). So, for the small-scale dynamo-generated field to saturate to subequipartition level by the processes described above, the galactic gas has to be predominantly neutral with an ion density less than $n_{i}^{c}$. Note that the value of $n_{i}^{c}$ is critically dependent on the turbulence parameters that obtain in the ISM of the galaxy, especially the turbulent velocity scale. For example for $V=5 \mathrm{kms}^{-1}$, and all other parameters as above, the critical density becomes $n_{i}^{c} \sim 0.5 \mathrm{~cm}^{-3}$; so even for an ionised hydrogen density as large as $10 \%-50 \%$ of a neutral density, taken here to be $1 \mathrm{~cm}^{-3}$ ), saturation could occur due to inefficient stretching.

Let us now ask what happens if the ion densities exceed the critical value $n_{i}^{c}$. In this case $\left(B_{p}^{2} / 8 \pi\right)<P_{\text {ext }}$ and the stretching constraint can only be satisfied only if the flux rope thickens further than the value implied by ambipolar drift, to a radius $r_{d}=R \sim l\left(C_{d} / 4 \pi\right)\left(\rho_{n} v_{l}^{2} /\left(2 P_{\text {ext }}\right)\right)$. If the flux ropes can thicken to this radius, the average energy magnetic energy density will be

$\frac{E_{B}(L)}{E_{T}} \sim N \frac{3 C_{d}^{2}}{128 \pi F} M_{T}^{2} \sim 7.3 \times 10^{-4} N C_{d}^{2}$

where $M_{T}$ is the Mach number of the turbulence and we have adopted the parameters $F \sim 4$ and $P_{g} / E_{T} \sim 1.7$, given above for the numerical estimate. The value of $\mathrm{N}$ should again be limited by the collapse of loops as discussed above.

However, it is not clear if flux ropes can thicken further than the radius implied by ambipolar drift, when dynamo action begins from weak seed fields. One possibility is that the magnetic pressure in the ropes acting on the fluid as a whole can thicken the rope. But this can only happen if the pressure in the rope becomes larger than the pressure outside, at least temporarily. Even if this were possible, as the flux rope thickens, flux freezing leads to a decrease of the field strength in the rope, and a consequent decrease in the thickening rate. The fluid pressure in the rope will also decrease as the rope expands. Note that this problem does not arise when ambipolar drift is causing the thickening. In a predominantly neutral medium, the ion pressure is much smaller than that due to neutrals. And the Lorentz force term in the Euler equation for the ions can be much larger than the ion pressure gradient term, and cause a relative drift of the ions with respect to the neutrals, carrying the field, and hence thickening the flux rope. 
If the ion density is larger than $n_{i}^{c}$ and the flux ropes cannot thicken sufficiently to resist stretching, then it is not clear how exactly the small scale field saturates. The field may get packed locally into a radius $\sim R$ by the folding motions associated with the turbulence, and then resist further stretching. On the other hand, the small-scale field may only saturate if the length of flux ropes, $N$, increases sufficiently to achieve equipartition with the turbulence. In either case the small-scale field will be highly intermittent. The effects of reconnection of this highly intermittent field (cf. Vishniac 1995, Lazarian and Vishniac 1996) could be important in deciding if the turbulence can still lead to large-scale dynamo action. In an interesting paper which came to our notice during the completion of the present work, Blackman (1996) discusses the possible effects of reconnection in greater detail, albiet in the case where the flux ropes are assumed to have a thickness $\sim R$, and assuming the gas is largely ionised.

\section{DISCUSSION AND CONCLUSIONS}

The large-scale galactic field is thought to be generated by a turbulent dynamo. However the same turbulence will produce magnetic noise at a more rapid rate. We have examined whether the Lorentz forces associated with the growing small-scale fields, can lead to their saturation, in a manner which preserves large-scale dynamo action. In doing this, we have also taken account of the ambipolar drift induced by the presence of a neutral component of the galactic gas.

The saturated state of the small-scale dynamo generated field, which we have motivated, does indeed preserve large-scale dynamo action. The crucial property of the smallscale dynamo generated field which allows this to happen is its spatial intermittency. The field can build up locally to a level which will lead to small-scale dynamo saturation, while at the same time having a sub-equipartition average energy density.

Numerical simulations of dynamo action due to mirrorsymmetric turbulence (Meneguzzi et al. 1981) or convection (Brandenburg et al. 1996) have indeed hinted at a saturated state of the small-scale dynamo as described above; a magnetic field concentrated into flux ropes, occupying a small fraction of the fluid volume, having peak fields comparable or in excess of equipartition value but average magnetic energy density only about $10 \%$ of the kinetic energy density.

We have described in section 4 and 5 the approach to this saturated state. As we noted in section 4, for conditions appropriate to galactic gas, the effective magnetic Reynolds number, even including ambipolar diffusion, is much larger than a critical value needed for small-scale dynamo action. However, in such a case, as the the small-scale field grows in strength, it continues to be concentrated into thin ropy structures, as in the kinematic regime. These flux ropes are curved on the turbulent eddy scales, while their thickness is set by the diffusive scale determined by the effective ambipolar diffusion. The growing magnetic tension associated with the curved flux ropes, acts to straighten them out. Frictional drag damps the magnetic energy associated with the wrinkle in the rope. Also, small-scale flux loops can collapse and disappear. These non-local effects operate on the eddy turnover time scale, when the peak field in a flux rope has grown to a few times the equipartion value. Their net effect is to make the random stretching needed for the small-scale dynamo inefficient and hence saturate the small-scale dynamo. However, the average energy density in the saturated small-scale field is sub equipartition, since it does not fill the volume. Such fields neither drain significant energy from the turbulence, nor convert eddy motion of the turbulence on the outer scale to wave-like motion. The diffusive effects needed for the large-scale dynamo operation are then preserved. This picture of small-scale dynamo saturation obtains when the ion density is less than a critical value of $n_{i}^{c} \sim 0.06-0.5 \mathrm{~cm}^{-3}\left(n_{n} / \mathrm{cm}^{-3}\right)^{2 / 3}$.

For very large ion densities $n_{i}>n_{i}^{c}$, the small-scale field is expected to saturate only when its energy density grows comparable to that of the turbulence. This is because, in this case the peak field, cannot grow sufficiently, (without its pressure exceeding the interstellar pressure), for the stretching constraint to apply. However, the spatial structure of the small-scale field, is still likely to be highly intermittent. The large-scale dynamo action will depend on how such a field responds to turbulent motions, especially whether the field can reconnect efficiently (cf. Vishniac 1995, Blackman 1996).

We discuss briefly, the implications of the above results for the origin of galactic fields. The viability of the saturation mechanism discussed here for limiting magnetic noise depends on the ionisation of the gas and the turbulence parameters. In the context of the Galaxy, a study of warm clouds by Spitzer and Fitzpatrick (1993) gives a range of electron densities for the clouds, with an average of $\sim 0.07 \mathrm{~cm}^{-3}$. They also deduce an average neutral density $\sim 0.2 \mathrm{~cm}^{-3}$. For such parameters, the magnetic noise will indeed saturate if the turbulent velocity is $\sim 5 \mathrm{kms}^{-1}$ and other parameters are as in Eq. (27).

In the case of a young galaxy with mass $\sim 10^{11} M_{\odot}$ that has just collapsed into a $10 k p c$ sized region, the average density is larger, and is $\sim 1 \mathrm{~cm}^{-3}$. The average column density for $H I$ is $\sim 10^{22} \mathrm{~cm}^{-2}$. The damped Ly- $\alpha$ systems seen in the spectra of high redshift quasars, and thought to be young galaxies do indeed have such HI column densities (cf. Wolfe 1995). The ionisation fraction is more uncertain. For such a high HI column density as inferred above, the gas is expected to become self shielded to external ionising flux. However, ionisation will result from UV emission from young stars embedded in the gas, whose importance depends on the uncertain star formation rates and stellar mass functions. From an observational point of view, a recent study of metal lines in these systems ( $\mathrm{Lu}$ et al., 1996) deduces an upper limit to their electron density, consistent with the average electron density in warm clouds in the Milky Way $\sim 0.07 \mathrm{~cm}^{-3}$ mentioned above. Further velocity widths deduced from $21 \mathrm{~cm}$ absorption due to neutral hydrogen in several damped Ly- $\alpha$ systems, also limit a turbulent component to the line width to be about $10 \mathrm{kms}^{-1}$ (cf. De Bruyn et al. , 1996). So, here also, the densities and turbulence parameters are expected to be in the range wherein the small-scale dynamo generated fields can saturate due to the tension forces, in a way which preserves large-scale dynamo action.

In our analysis, so far, we have ignored the small-scale field generated by the tangling of the large-scale field by the turbulence. Even when the dynamo generated small scale field has saturated, this will provide an additional source 
of small-scale magnetic noise. As the large-scale field grows, so does this component of the small-scale field with an energy density ultimately decided by the nature of the MHD turbulence (cf. Zeldovich et al. 1983). We hope to return to this issue in a later work. One then expects two components to the small-scale magnetic field in the interstellar medium of a galaxy. First, a ropy, intermittent component, with flux ropes curved on scale $L \sim 100 p c$, say, and thickness $r_{d} \sim 10^{-2} L \sim 1 p c$, with peak field a few times equipartition. Second, a more diffuse small-scale field related in strength to the large-scale field. It would be interesting to search for both of these components in the interstellar medium of galaxies.

The effect of ambipolar drift, together with the smallscale dynamo, can also influence galactic magnetic field generation, indirectly, in another fashion. Note that any dynamo needs a seed field to act upon. Since the small-scale dynamo acts to generate fields more rapidly than the large-scale dynamo, the magnetic noise so generated may itself provide a significant seed for the large-scale dynamo (cf. Beck et al. 1994). However the small-scale dynamo also leads to highly ropy fields with a rope thickness, $r_{d}$. In a fully ionised gas $r_{d}=r_{d}^{i} \sim L / R_{m}^{1 / 2}$ will be very small, since $R_{m}>>1$. The overlap of such a field with a large-scale dynamo eigenfunction will be small. However, if small-scale dynamo action proceeds in the presence of neutrals, $r_{d}=r_{d}^{n} \sim L / R_{a m b i}^{1 / 2}>>$ $r_{d}^{i}$ in general, since $R_{a m b i}<<R_{m}$. So, when neutrals are present, the small-scale dynamo-generated magnetic noise will provide a more coherent seed field, for large-scale dynamo action. This will act to shorten the timescale for the generation of large-scale galactic magnetic field, to the microgauss level, at higher redshift.

Note that, after recombination, the residual ionisation fraction of the intergalactic medium drops to about $10^{-4}$ $10^{-5}$. One may be tempted to apply some of the results obtained here to the first generation of objects which collapse at high redshifts. The limitations of our semi-quantitative arguments, and our assumption of a homogeneous galactic interstellar medium, in reaching the above conclusions, needs little emphasising. It would also be fruitful to find ways of incorporating, more fully, the dynamics of the velocity correlations, as we have done for the magnetic correlations. This full MHD turbulence problem appears formidable at present. Nevertheless, the results obtained here encourage the belief that that the turbulent galactic dynamo could indeed be made to produce large-scale fields, in the presence of a significant neutral component.

\section{ACKNOWLEDGMENTS}

At Sussex KS is supported by a PPARC Visiting Fellowship. He thanks John Barrow for invitation to Sussex and both him and Leon Mestel for their detailed comments on the manuscript. At Princeton KS was supported by NSF grant AST-9424416. Partial travel support came from IAU Commission 38. He thanks Jerry Ostriker and Ed Turner for the invitation to visit Princeton University Observatory, and the staff there for a warm reception. Discussions with collegues too numerous to mention have been very helpful, during the course of this work.

\section{REFERENCES}

Beck, R., Brandenburg, A., Moss, D., Shukurov, A. \& Sokoloff, D., 1996, Ann. Rev. Astron. Astrophys., 34, 155.

Beck, R., Poezd, A.D., Shukurov, A. \& Sokoloff, D., 1994.. A \& A, 289, 94.

Brandenburg, A., 1994, Lectures on Solar and Planetary Dynamos, ed. Proctor, M. R. E. \& Gilbert, A. D., Cambridge University press, p117.

Brandenburg, A., Jennings, R. L., Nordlund, A., Rieutord, M., stein, R. F. \& Touminen, I., 1996. JFM, 306, 325.

Blackman, E., 1996. Phys. Rev. Lett., 77, 2694.

Chandran, B., 1996. Princeton University Observatory preprint POPe-659.

Cattaneo, F. \& Vainshtein, S. I., 1991. ApJ, 376, L21.

De Luca, E. E., Fisher, G. H. \& Patten, B. M., 1993. ApJ, 411, 383.

De Bruyn, A. G., O'Dea, C. P. \& Baum, S. A., 1996. A \& A, 305, 450.

Draine, B. T., 1980. ApJ, 241, 1021.

Draine, B. T., 1986. MNRAS, 220, 133.

Field, G. B., 1996. The Physics of the Interstellar Medium and Intergalactic Medium, ed. Ferrara et al., ASP Conf. Ser., 80, p1.

Kazantsev, A. P., 1968. Sov. Phys. JETP, 26, 1031.

Kleeorin, N. I., Ruzmaikin, A. A., Sokoloff, D. D., 1986. Plasma Astrophysics, ESA Publication, p557.

Krause, F. \& Radler, K.-H., 1980. Mean-Filed Magnetohydrodynamics and Dynamo Theory, Pergamon press, Oxford.

Kulsrud, R.M. \& Anderson, S.W., 1992. ApJ., 396, 606.

Landau, L. \& Lifshitz, E. M., 1987. Fluid Mechanics, Pergamon Press, Oxford.

Lazarian, A. \& Vishniac, E., 1996. Polarimetry of the Interstellar medium, ASP Conf. Ser., 97, p537.

Lu, L., Sargent, W. L. W., Barlow, T. A., Churchill, C. W. \& Vogt, S. S. 1996, ApJ Suppl., 107, 475.

Meneguzzi, M., Frisch, U. \& Pouquet, A., 1981. Phys. Rev. Lett., 47,1060

Mestel, L. \& Spitzer, L., 1956. MNRAS, 116, 503.

Mestel, L. \& Subramanian, K., 1991. MNRAS, 248, 677.

Moffat, H. K., 1978. Magnetic Field Generation in Electrically Conducting Fluids, Cambridge University Press, Cambridge.

Parker, E. N., 1979. Cosmic Magnetic Fields, Clarendon press, Oxford.

Ratra, B., 1992. ApJ Lett., 391, L1.

Rees, M. J., 1987. Quart. J.R.A.S., 28, 197.

Rees, M.J., 1994. Cosmical Magnetism, ed. Lynden-Bell, D., Kluwer, London, p155

Ruzmaikin, A. A., Shukurov, A. M. \& Sokoloff, D. D., 1988. Magnetic Fields of Galaxies, Kluwer, Dordrecht.

Ruzmaikin, A. A., Shukurov, A. M. \& Sokoloff, D. D., 1989. MNRAS, 241, 1.

Spitzer, L., 1978. Physical Processes in the Interstellar medium, Wiley-Interscience, New York.

Spitzer, L. \& Fitzpatrick, E., 1993. ApJ, 409, 299.

Subramanian, K., 1995. Bull. Astr. Soc. Ind., 23, 481.

Subramanian, K., 1997. in Preparation (Paper II).

Subramanian, K., Narasimha, D. \& Chitre, S.M., 1994. MNRAS. 271, L15.

Vainshtein, S. \& Kichatinov, L. L., 1986, J. Fluid. Mech., 168, 73.

Vainshtein, S. \& Rosner, R., 1991, ApJ., 376, 199.

Vishniac, E. T., 1995. ApJ, 446, 724.

Wolfe, A. M., 1995. QSO Absorption Lines, ed. Meylan G., Springer, Berlin, p13.

Zeldovich, Ya.B., Ruzmaikin,A.A. \& Sokoloff,D.D., 1983. Magnetic fields in Astrophysics, Gordon and Breach, New York.

Zweibel, E. G., 1986. ApJ, 329, 384. 
This paper has been produced using the Royal Astronomical Society/Blackwell Science $\mathrm{AT}_{\mathrm{E}} \mathrm{X}$ style file. 